\title{
Debates electorales en los VOD de las RTV Públicas de Europa
}

\section{Electoral debates in the VODs of the Public RTVs of Europe}

\author{
Martín Vaz-Álvarez. Universidad de Santiago de Compostela. España. \\ martin.vaz.alvarez@usc.es \\ $[\mathrm{CV}](\mathrm{C})$ \\ José Miguel Túñez-López. Universidad de Santiago de Compostela. España. \\ miguel.tunez@usc.es
}

$[\mathrm{CV}]$ (C)

Carmen Costa-Sánchez. Universidad de la Coruña. España.

carmen.costa@udc.es

$[\underline{\mathrm{CV}}]$ (1)

Este artículo forma parte de las actividades del proyecto de investigación (RTI2018-096065-B-I00) del Programa Estatal de I $+D+i$ orientado a los Retos de la Sociedad del Ministerio de Ciencia, Innovación y Universidades y del Fondo Europeo de Desarrollo Regional (FEDER) sobre Nuevos valores, gobernanza, financiación y servicios audiovisuales públicos para la sociedad de Internet: contrastes europeos y españoles. El autor Martín Vaz Álvarez es beneficiario de una ayuda de Formación de Profesorado Universitario del Ministerio de Ciencia, Innovación y Universidades de España (ref. FPU19/06204).

\section{Cómo citar este artículo / Referencia normalizada}

Vaz-Álvarez, M., Túñez-López, J. M. y Costa-Sánchez, C. (2020). Debates electorales en los VOD de las RTV Públicas de Europa. Revista Latina de Comunicación Social, (76), 81-96. https://www.doi.org/10.4185/RLCS-2020-1438

\begin{abstract}
RESUMEN
Introducción: En los medios públicos, el debate electoral es de especial interés por las reglas que deben adoptar estas televisiones para cumplir con sus compromisos de servicio público y mantener los valores de diversidad, universalidad y participación. En el nuevo escenario que plantean las plataformas propias de VOD la inclusión de estos debates electorales televisados y las variaciones en su formato se plantea como un ejercicio de transparencia y un compromiso con la memoria audiovisual del país que refuerza los valores de diversidad, proximidad y universalidad representativa. Metodología: El artículo revisa de un modo comparativo el uso de este formato en siete de las principales emisoras públicas de televisión de Europa siguiendo los modelos de Hallin y Mancini y con una subdivisión regional dentro del modelo democrático-corporatista para obtener resultados con un corte étnico-político. Resultados y conclusiones: Existe una notable disparidad entre las televisiones públicas que tienen accesibles los debates electorales en sus plataformas de VOD. El análisis desvela que, a pesar de su trascendencia, los debates no se encuentran disponibles en los entornos online del medio, aunque sí son accesibles desde plataformas ajenas, principalmente YouTube.
\end{abstract}

PALABRAS CLAVE: plataformas de vídeo por demanda, debates electorales, RTV pública, VOD, participación, nuevos formatos, Europa. 


\begin{abstract}
Introduction: In the public media, the electoral debate is of special interest because of the rules that these televisions must adopt to fulfill their public service commitments and maintain the values of diversity, universality, and participation. In the new scenario posed by VOD's platforms, the inclusion of these televised electoral debates and variations in their format are presented as an exercise of transparency and a commitment to the country's audiovisual memory that reinforces the values of diversity, proximity, and representative universality. Methodology: The article comparatively reviews the use of this format in seven of the main public television stations in Europe following the models of Hallin and Mancini and with a regional subdivision within the democraticcorporatist model to obtain results with an ethnical-political cut. Results and conclusions: There is a notable disparity between public televisions that have access to electoral debates on their VOD platforms. The analysis reveals that, despite its importance, the debates are not available in the online media environments, although they are accessible from third-party platforms, mainly YouTube.
\end{abstract}

KEYWORDS: VOD platforms, electoral debates, public television, VOD, participation, new formats, Europe.

\title{
CONTENIDOS
}

1. El creciente rol de las plataformas de VOD, 2. Los debates electorales en televisión, 3. Metodología y preguntas de investigación, 4. Análisis de número de debates y formatos en Europa, 4.1. Andorra, 4.2. Alemania, 4.3. Austria, 4.4. Bélgica, 4.5. Holanda, 4.6. Reino Unido, 4.7. España, 4.8. Análisis por modelos, 5. Discusión, 6. Referencias.

\section{EI creciente rol de las plataformas de VOD}

Las audiencias de todo el mundo han adaptado en los últimos años nuevas formas de consumo de contenidos con el florecimiento de los servicios de VOD (Video on Demand) (IHS Markit, 2019) y las automatizaciones algorítmicas (Túñez, Toural, Valdiviezo-Abad, 2019) impulsadas entre otras cosas por la proliferación de SmartTVs (Ofcom, 2018). Particularmente en Europa, se observa cómo los espectadores pasan cada vez más tiempo viendo la televisión no lineal (gratuita, por suscripción, vídeo a la carta y vídeo transaccional a la carta), y cada vez menos tiempo viendo la televisión lineal tradicional (Ofcom, 2018, Consejo de Europa, 2019).

Aunque se ha producido un aumento general de los minutos por día por persona que pasa viendo contenidos audiovisuales, en países como el Reino Unido, Italia y España, los servicios de vídeo a la carta han reducido los minutos que se dedican a ver la televisión tradicional (IHS Markit, 2019). Se espera que esta tendencia aumente en los próximos años, ya que nuevos competidores como Apple y Disney, entre otros, entrarán en el mercado a finales de 2019 y a lo largo de 2020 (Consejo de Europa, 2019).

Este incremento del consumo televisivo plantea dos retos para los radiodifusores de servicio público europeos. Por un lado, los rápidos cambios en los hábitos de la audiencia les obligarán a desarrollar estrategias de contenidos cada vez más avanzadas y orientadas al VOD para retener a las audiencias en movimiento. Por otro, a medida que se intensifica la lucha por la atención del espectador, con las plataformas de suscripción de crecimiento exponencial como Netflix, HBO o Amazon y las próximas Disney+ o AppleTV, los radiodifusores de servicio público tendrán que dedicar una atención especial al desarrollo de sus plataformas de VOD. Además, les exige organizar y presentar sus contenidos de manera que estén siempre disponibles, no solo para competir con otras plataformas, sino también, en el caso de los medios de servicio público, para generar en sí mismos una hemeroteca audiovisual con valor histórico y social. 
El compromiso de los algoritmos y las plataformas de VOD con los valores de servicio público ya ha sido objeto de investigación a nivel internacional, pero más intensamente en el contexto europeo. Uno de los conflictos más comunes cuando se evalúa la justificación de los sistemas de recomendación es el principio de participación. Aunque el engagement es clave para mantener la relevancia, la mayoría de los académicos están de acuerdo en que esto no debe ocurrir a cualquier costo.

Siguiendo la pauta sugerida por John Reith sobre "dar al público lo que quiere aunque no sepa que lo quiere" (Hutchinson, Sorensen, 2018; Fields, Jones, Cowlishaw, 2018), los sistemas de recomendación deben tener en cuenta las nociones de universalidad, diversidad, transparencia e imparcialidad (Fields, Jones, Cowlishaw, 2018); Moe, Van den Bulck, 2017; Hutchinson, Sorensen, 2018), que son fundamentales para reforzar la legitimidad del PSM (Campos-Freire, RodríguezCastro, de Mateo-Pérez, 2019).

En otras palabras, aunque se orientan las relaciones con las audiencias a través de recomendadores de contenidos, los medios públicos no pueden dejar de cumplir con su misión y sus compromisos derivados directamente de ese carácter de titularidad pública, ya que no hacerlo comprometería su compromiso con la sociedad y podría exponer a la audiencia a contenidos unilaterales o 'bubble filtered' basados exclusivamente en sus propios patrones de consumo o contenidos predefinidos como "agradables" (Pariser, 2011).

Además, en un momento en el que los VODs también se postulan como elementos co-creativos, en tanto que toman en cuenta las preferencias y gustos de la audiencia para generar recomendaciones (Fields, Jones, Cowlishaw, 2018), y que estas después repercuten en la promoción de unos u otros contenidos por parte de la plataforma, estos mecanismos tienen la oportunidad de generar espacios de co-creación y participación ciudadana, que refuercen el valor de innovación identificado por la EBU para las televisiones públicas, y que sigue la línea de otros académicos sobre el valor de la participación en la gestión pública (Simonofski, Snoeck, Vanderose, 2019; Alford, Hughes, 2008).

\section{Los debates electorales en televisión}

Los debates como género, de un modo general, y los debates en campañas electorales han generado innumerables investigaciones académicas. Se han analizado todas las vertientes. Fijando la mirada del investigador en el formato (Kraus,1962; Kraus 1988; Carlin, Howard, Stanfield y Reynolds, 1991; McKinney y Carlin, 2004; Schroeder, 2008; Schroeder 2012; Turcotte, 2015), la comparativa del formato y su uso en países diferentes (Schroeder, 2012; Micovic, 2014), el formato como elemento modernizador de campañas (Campus, 2010; Caprara, 2007; Berrocal, 2003: 55-79; Langer, 2007: 371-387), los contenidos discursivos con estudios de la puesta en escena, la realización, la retórica (Matera y Salwen, 1996; Anaya, 2014), la pragmática (Bañon, 1997; Fernández , 2000; Blas, 2001; Fuentes, 2009; Blas, 2011; Micovic, 2014) o el papel de los periodistas (Martel, 1983; Stepp, 1987; Lanoue y Schrott, 1991; Coleman, 2000; Kraus, 2000; Schroeder, 2016; Núñez-Mussa, 2018).

En España destacan, entre otras, las aportaciones al análisis de los debates televisados realizadas por Díez Nicolás y Semetko, 1995; Vidal Riera, 1997; Berrocal, Dader y Rospir, 2003; Marín, 2003; Canel, 2006; Castells, 2009; Gallego Reguera, 2009; Ruiz Contreras, 2007; Campo Vidal, 2013; Gallego Reguera, 2011; Gallego Reguera, 2012; Conde-Vázquez, Fontenla-Pedreira y Rúas-Araújo, 2019; y Gallego Reguera y Martínez, 2019. 
Resulta muy ilustrativa la investigación de Carolina Herranz-Rubio sobre la producción científica en torno a debates electorales en España. Su análisis bibliométrico de artículos científicos, capítulos de libro y los libros, actas de congresos y las tesis publicados en el periodo 1993-2018 desvela que predominan, por orden decreciente, el análisis de los contenidos, de la cobertura mediática, de los efectos de los debates, del estudio de los formatos, de la organización, de la historia, la legislación de estos y el género del debate. (2020, p. 11). La autora destaca (2020, p. 15) que mientras las investigaciones en EEUU se orientan a los efectos sobre el voto o en Francia a los aspectos lingüísticos, en España se ha orientado al estudio de los contenidos y de la cobertura. Los datos que aporta Herranz-Rubio señalan hacia un análisis de los contenidos de los debates desde una perspectiva pragmática en más de la mitad de los documentos analizados (el 51,2\%) seguido de estudios de la Retórica (30,2\%).

Dentro de las teorías pragmáticas, el estudio de la descortesía ha sido el que más ha interesado a

los investigadores (con un 25,6\%). En segundo lugar, se encuentra el análisis de las preguntas, es decir, lo relativo a su naturaleza, sus funciones, sus tipos, etc. (con el 7,0\%). En cuanto a la Retórica, destaca el estudio de las estrategias discursivas (con el 9,4\%). En relación a la Lingüística, la comunicación no verbal ha sido el aspecto más investigado $\mathrm{y}$, dentro de esta, ha destacado el estudio de los gestos y las posturas (con el 7,0\%). (2020, p. 13).

No se han encontrado en la literatura científica referencias de investigaciones sobre la relación de los debates electorales y los VOD's. En la revisión bibliográfica tampoco se han encontrado artículos orientados a analizar comparativamente el uso del formato en las televisiones públicas. Este artículo resulta, pues, una propuesta oportuna ya que revisa ambas cuestiones al proponer un recuento de debates electorales televisados y su registro en las plataformas propias de cada emisora pública o en otros soportes, principalmente, en canales promovidos por actores colectivos en las redes sociales.

\section{Metodología y preguntas de investigación}

Esta investigación toma como punto de partida que la presencia de debates electorales en la carta de contenidos de estas plataformas 'On Demand' debería formar parte de la labor del medio de servicio público de proporcionarle al espectador la capacidad de recuperar y revisar aquellos contenidos que forman parte de las campañas políticas que terminan por la elección de sus representantes, además de recoger estos vídeos como parte de un repositorio de la historia política y la memoria audiovisual del país. Y más aun teniendo en cuenta que el debate no es un producto para efímero para consumo puntual sino que su contenido marca referencias para evaluar las actuaciones posteriores de los participantes a lo largo de la legislatura.

Ante la ausencia de referencias previas de trabajos similares se proyecta el estudio como una investigación deductiva, de carácter exploratorio y descriptivo. El objetivo principal es definir la posición de los debates electorales televisados en el contexto de las plataformas de VOD de las televisiones públicas para identificar si estas plataformas se están utilizando también como hemeroteca de la memoria audiovisual política de los países y como elemento de transparencia y acceso de los ciudadanos a las discusiones políticas más relevantes de cara a la elección de sus representantes. Además, se pretende, a modo de objetivo secundario, saber si aportar una visión actualizada de los formatos que se usan en los debates electorales televisados en medios de servicio público. 
Las preguntas planteadas para conseguir los objetivos propuestos en este artículo son las siguientes:

PI-1. ¿Cuántos debates electorales se ha realizado o emitido en los medios públicos de cada país a lo largo de los últimos años?

$P I-2$. ¿Ha variado el formato en cuanto a número de partidos participantes?

PI-3. ¿Recogen las plataformas de VOD de las RTV públicas europeas los debates electorales televisados emitidos en sus propios canales?

PI-4. ¿Cuáles son las tendencias en los formatos de los debates electorales televisados en las RTV públicas de Europa?

PI-5. ¿Existen diferencias o similitudes en estos formatos según el modelo de RTV pública?

PI-6. ¿Existen rasgos comunes o diferencias en el formato de los debates electorales televisados según los 3 modelos de Hallin y Mancini (2004)?

Para la realización de este artículo se han seleccionado una muestra intencional no probabilística. Se han tomado como objeto de estudio siete radiotelevisiones públicas europeas repartidas de modo equilibrado siguiendo los modelos de Hallin y Mancini y con la particularidad de una subdivisión regional dentro del modelo democrático-corporativista para obtener resultados con un corte étnicopolítico. Los medios objeto de estudio son los siguientes:

- Modelo pluralista polarizado: Andorra (Andorra Difussió) y España (RTVE).

- Modelo democrático-corporatista: Alemania (ZDF), Austria (ORF).

- Subdivisión regional: Holanda (NPO) y Bélgica (VRT).

- Modelo liberal: Reino Unido (BBC).

En todos los casos se ha procedido a realizar una búsqueda preliminar sobre todas las elecciones federales o generales al Parlamento de cada uno de los siete países en el periodo 2009-2019, ambos incluidos. Después de identificar los años y el número de elecciones acontecidas en ese período, se ha procedido a la búsqueda pormenorizada de todos los vídeos referentes a las elecciones en cada uno de los países, tanto en sus propias plataformas de VOD, como en YouTube, Vimeo y en búsquedas generales por buscador web. Para realizar esta búsqueda en cada uno de los países primero fue necesario identificar las palabras con las que identificaban los contenidos relativos a las elecciones en su propio idioma, para obtener resultados más precisos en cada uno de los países. Una vez identificados y recogidos todos los debates electorales televisados disponibles en las plataformas online, se procedió al análisis de su formato y la identificación del número de participantes a través de una ficha de recogida de datos de elaboración propia, testada antes de realizar la recogida de datos. El rastreo on line se desarrolló en los meses de septiembre y principios de octubre de 2019. La interpretación de datos se desarrolló a lo largo del mes de octubre de 2019. Se ampliaron los resultados en noviembre para incorporar el debate de las elecciones generales del 10 de noviembre de 2019 en España.

\section{Análisis de número de debates y formatos en Europa}

A continuación se presentan los resultados compilados en tablas resumen en las que contabiliza el número de debates y su formato de los 7 países europeos incluidos en la muestra. Andorra y España representan en este caso el modelo mediterráneo pluralista polarizado, Alemania y Austria el modelo democrático-corporatista, complementado por Bélgica y Holanda, comparando las posibles similitudes o diferencias regionales existentes dentro de este mismo modelo y, por último, la BBC del Reino Unido para el modelo liberal. 


\subsection{Andorra}

Tabla 1. Debates en Andorra Difussió Elecciones Parlamentarias.

\begin{tabular}{|c|c|c|c|}
\hline \multicolumn{2}{|c|}{ Modelo mediterráneo pluralista polarizado } & 1 & 1 \\
\hline Número & 0 & 2015 & 2019 \\
\hline Año & 2011 & 4 & 7 \\
\hline Número de partidos & NA & Sí & Sí \\
\hline Accesible en el VOD & NA & Sí & Sí \\
\hline Accesible online & NA & \\
\hline
\end{tabular}

Fuente: elaboración propia.

Andorra celebró debates electorales televisados durante las elecciones parlamentarias de los años 2015 y 2019. Además de debates con los representantes de los partidos aspirantes al gobierno de Andorra, realizó otros sub-debates regionales para tratar asuntos de índole local.

El número de integrantes en los debates de Andorra Diffusió casi se duplicó en 2019 ya que aumentó de 4 en el año 2015 a 7 en las últimas elecciones, adaptando su formato a la nueva realidad política que se presentaba en su país. Además, en la propia página web de la corporación existen segmentos dedicados y permanentemente accesibles sobre la cobertura de cada una de las elecciones, en donde se incluyen todo tipo de noticias relacionadas con las elecciones y también los diferentes debates que se realizaron en el canal.

\subsection{Alemania}

Tabla 2. Debates en ZDF Elecciones Federales.

\begin{tabular}{|c|c|c|c|}
\hline \multicolumn{2}{|c|}{ Modelo democrático-corporatista } \\
\hline Número & 1 & 1 & 1 \\
\hline Año & 2009 & 2013 & 2017 \\
\hline Número de partidos & 2 & 2 & 2 \\
\hline Accesible en el VOD & No & No & Sí \\
\hline Accesible online & Sí & Sí & \\
\hline
\end{tabular}

Fuente: elaboración propia.

El 27 de septiembre de 2009 se celebraron elecciones federales para elegir a los miembros del $17^{\circ}$ Bundestag de Alemania, donde los resultados preliminares mostraron una victoria de la Unión Democrática Cristiana (CDU), su partido hermano bávaro, la Unión Social Cristiana (CSU), y el Partido Democrático Libre (FDP).

El debate electoral en este caso tuvo lugar entre Angela Merkel, de la CDU, contra su principal oponente, Frank-Walter Steinmeier, del Partido Socialdemócrata (SPD). 
El mismo modelo se repitió posteriormente, en el año 2013, con un debate a dos entre Angela Merkel y Peer Steinbrück, y, por última vez, en 2017 también entre Angela Merkel y el socialista Martin Schulz.

La Mediathek de la ZDF (segundo canal público donde se hacen los debates de las elecciones federales a 2) solo presenta el vídeo del último debate realizado en el país (2017) así como otros contenidos de interés alrededor del mismo tema como el pre-debate o el post-debate. La MediathekZDF fue 'relanzada' en el año 2016, motivo por el cual las grabaciones de los debates previos a esa época no se encuentren disponibles en la plataforma. Eso indica, sin embargo, que no se ha hecho trabajo de recuperación de estos archivos para ser incluidos en la hemeroteca. En YouTube, sin embargo, sí es posible acceder a estos contenidos.

\subsection{Austria}

Tabla 3. Debates en ORF Elecciones Federales.

\begin{tabular}{|c|c|c|c|}
\hline \multicolumn{2}{|c|}{ Modelo democrático-corporativista } & 10 \\
\hline Número & 10 & 2017 & 2019 \\
\hline Número de partidos & 2013 & 2 & 2 \\
\hline Accesible en el VOD & 2 & No & No \\
\hline Accesible online & No & Sí & Sí \\
\hline
\end{tabular}

Fuente: elaboración propia.

El formato de debates electorales de la televisión austríaca es similar al de la televisión pública alemana. Se trata de un formato de 'duelo' entre dos participantes. A diferencia del modelo alemán (en el que solo se enfrentan los dos candidatos con opciones reales de ganar) en Austria se celebran en total 10 debates en cada elección, haciendo todas las combinaciones de parejas políticas posibles, es decir, que cada uno de los representantes de los partidos debate en un duelo a 2 con cada uno de todos los otros representantes.

Ninguno de estos duelos, sin embargo, se encuentran disponibles en la plataforma de VOD de la cadena pública austriaca ORF, en la que, sin embargo, sí que podemos encontrar algunos debates alemanes y debates históricos austríacos.

\subsection{Bélgica}

Tabla 4. Debates en VRT Elecciones Federales.

\begin{tabular}{|c|c|c|c|}
\hline \multicolumn{3}{|c|}{ Modelo democrático-corporativista (subdivisión regional) } \\
\hline Número & NA & 1 & 1 \\
\hline Año & 2010 & 2014 & 2019 \\
\hline Número de partidos & 0 & 2 & 6 \\
\hline
\end{tabular}


RLCS, Revista Latina de Comunicación Social, 76, 81-96

[Investigación] DOI: 10.4185/RLCS-2020-1438 | ISSN 1138-5820 | Año 2020

\begin{tabular}{|c|c|c|c|}
\hline \hline Accesible en el VOD & No & No & No \\
\hline Accesible online & No & Sí & Sí \\
\hline
\end{tabular}

Fuente: elaboración propia.

Los debates emitidos por la VRT han pasado de 0 en 2010 a 6 en 2019, año en el que aparecen nombrados en su página web oficial, a pesar de que el vídeo no se encuentra disponible. Un mensaje en la página web hace entender que en un momento determinado sí lo estuvo, pero que este vídeo fue retirado posteriormente.

El primer debate televisado en la VRT belga disponible online se corresponden con el celebrado durante las elecciones federales de 2014, las cuales se corresponden con la mayor operación electoral llevada a cabo en el país, escogiendo no solamente al nuevo parlamento belga, sino también a las asambleas descentralizadas y los miembros del parlamento europeo.

La VRT, a pesar de la importancia de estas elecciones en el contexto Belga, no tiene disponibles estos vídeos en su página web, aunque sí se pueden encontrar, en cambio, en otras plataformas como YouTube, subidos por usuarios ajenos a la corporación.

\subsection{Holanda}

Tabla 5. Debates en al NPO. Elecciones Generales.

\begin{tabular}{|c|c|c|c|}
\hline \multicolumn{2}{|c|}{ Modelo democrático-corporativista (subdivisión regional) } \\
\hline Número & 1 & 1 & 1 \\
\hline Año & 2010 & 2012 & 2017 \\
\hline Número de partidos & 4 & 8 & 14 \\
\hline Accesible en el VOD & No & No & Sí \\
\hline Accesible online & Sí & Sí & Sí \\
\hline
\end{tabular}

Fuente: elaboración propia.

En Holanda, los participantes en el debate más reciente son los 14 partidos más grandes según la Guía de votación del 1 de febrero de 2017 y la distribución actual de escaños en la Cámara baja. Los seis partidos más pequeños debaten en tríos, mientras que los líderes de los ocho partidos más grandes entran en discusiones 1 a 1 .

Este diseño se determinó el 2 de febrero de manera colaborativa entre la audiencia y la televisión a través de un sorteo a través de NOS en Facebook y el canal temático NPO News. También se celebra en la NPO holandesa un debate con dos escenarios: local y nacional, en el cual se discuten temas como la atención sanitaria y el empleo. Se utilizan dos platós para este debate, uno en el cual están los representantes locales y en otro los nacionales.

El número de partidos y el formato de los debates fue cambiando a lo largo del tiempo, si bien en 2010 el debate consistió tan solo en un debate a 4 entre los principales partidos candidatos a presidir 
el ejecutivo holandés, en 2012 se instaura por primera vez el formato de 'duelos múltiples' con 8 candidatos (4 y 4 repartidos en dos mesas).

Tan solo el último de estos debates, el correspondiente a las elecciones del año 2017, está disponible en la plataforma de VOD de la NPO holandesa, en la cual se entiende que aún no se ha hecho una recuperación de archivos para incluir en la hemeroteca, salvo aquellos especialmente relevantes por históricos. Los vídeos de debates anteriores sin embargo sí que están disponibles en YouTube, pero subidos por otros usuarios.

\subsection{Reino Unido}

Tabla 6. Debates en la BBC Elecciones Generales.

\begin{tabular}{|c|c|c|c|}
\hline \multicolumn{2}{|c|}{ Modelo liberal } \\
\hline Número & 3 & 4 & 3 \\
\hline Año & 2010 & 2015 & 2017 \\
\hline Número de partidos & $7-7-3$ & 7 y 2 & $7-7-2$ \\
\hline Accesible en el VOD & No & No & No \\
\hline Accesible online & Sí & Sí & Sí \\
\hline
\end{tabular}

Fuente: elaboración propia.

Los debates de las elecciones generales del Reino Unido de 2010 consistieron en una serie de tres debates entre los líderes de los tres partidos principales que disputaron las elecciones generales de 2010: Gordon Brown, primer ministro y líder del Partido Laborista; David Cameron, líder de la oposición y el partido conservador; y Nick Clegg, líder del tercer partido político más grande del Reino Unido, los Demócratas Liberales. Fueron los primeros debates de este tipo que se transmitieron en vivo en el período previo a las elecciones en el Reino Unido.

Tan solo uno de estos tres debates ocurrió en televisión púbica. Todos los debates se desarrollaron sin interrupción durante 90 minutos y fueron transmitidos semanalmente por ITV, Sky y la BBC. La primera mitad de cada debate se centró en un tema particular (asuntos internos, internacionales y económicos), antes de discutir cuestiones generales. Las preguntas no fueron reveladas a los líderes antes del debate.

Además de los debates de los líderes, también los portavoces financieros de los tres partidos principales participaron en un debate centrado en la economía, con el canciller del Tesoro, Alistair Darling, debatiendo con el ex-canciller George Osborne y el portavoz del Tesoro de los Demócratas Liberales, Vince Cable en Channel 4. Los debates también tuvieron lugar entre el 19 de abril y el 5 de mayo, una serie de debates también tuvieron lugar en la serie de televisión política de la BBC The Daily Politics, entre miembros del partido Laborista y sus homólogos Conservadores, Liberales Demócratas y representantes de Green Party, Scottish National Party, Plaid Cymru y UK Independence Party.

En el año 2015 el primero de los debates fue un programa uno a uno entre David Cameron, Primer Ministro (Partido Conservador), y Ed Miliband, Líder de la Oposición (Partido Laborista). El 
segundo contó con Cameron, Miliband, Nick Clegg, Viceprimer Ministro (Demócratas Liberales), Nigel Farage (UKIP), Natalie Bennett (Partido Verde de Inglaterra y Gales), Nicola Sturgeon, Primer Ministro de Escocia (SNP) y Leanne Wood (Plaid Cymru). El tercer debate contó con los líderes de los cinco partidos de oposición: Miliband, Farage, Bennett, Sturgeon y Wood. En el último de los debates, los participantes fueron Cameron, Miliband y Clegg. Solo Miliband del Partido Laborista participó en los cuatro eventos.

En el año 2017 se produjeron 2 debates a 7, sin contar con la presencia de los dos líderes con mayores posibilidades de gobierno (Theresa May y Jeremy Corbyn, en este caso). En una repetición del formato empleado antes de las elecciones de 2015, Jeremy Corbyn y Theresa May fueron posteriormente entrevistados por Jeremy Paxman, de Channel 4 frente a una audiencia de estudio en directo.

Ninguno de los debates que tuvieron lugar en el Reino Unido durante las 3 pasadas elecciones están disponibles en su VOD. De hecho, al intentar buscar dentro de esa plataforma contenidos relacionados con las elecciones, nos encontramos con que a pesar de aparecer nombrados en la plataforma, no están disponibles para su visualización.

\subsection{España}

Tabla 6. Debates en RTVE Elecciones Generales.

\begin{tabular}{|c|c|c|c|}
\hline \multicolumn{2}{|c|}{ Modelo mediterráneo pluralista polarizado } \\
\hline Número & 2 & 1 & 2 \\
\hline Año & 2015 & 2016 & 2019 \\
\hline Número de partidos & 9 y 2 & 4 & 4 y 5 \\
\hline Accesible en el VOD & Sí & Sí & Sí \\
\hline Accesible online & Sí & Sí & Sí \\
\hline
\end{tabular}

Fuente: elaboración propia.

En el año 2015, en España se probó un formato de debate sin precedentes hasta el momento, un debate a 9 entre los partidos con representación parlamentaria y en el que también estuvieron Podemos y Ciudadanos, los dos partidos emergentes, que, en ese momento según las encuestas, tenían opciones de entrar en la próxima legislatura en el Congreso. Posteriormente, se celebró un debate entre los dos principales candidatos a la presidencia del gobierno, Pedro Sánchez y Mariano Rajoy, en un clásico debate a dos que había sido la norma hasta el anterior debate a 9.

Ya en el año 2016, el formato del debate cambió para incluir a los dos partidos emergentes que ya habían consolidado su presencia en el Congreso: Ciudadanos y Podemos. El planteamiento de este debate en cuanto a formato no varió demasiado más allá del número de participantes, siguió estructurándose por bloques temáticos y con un solo moderador.

Posteriormente, en el año 2019, tuvimos la oportunidad de presenciar 2 debates en elecciones generales (uno por cada elección). En este caso el número de participantes varió de 4 en el primero, a 5 en el segundo, con la irrupción de VOX en el congreso. El esquema por el que se guió el debate siguió los bloques temáticos ya marcados en anteriores ocasiones, con la salvedad de que esta vez hubo dos moderadores, uno representando a la televisión pública y otro a un canal comercial. 
Todos los vídeos de los debates, tanto de las elecciones de 2015, 2016 y las dos de 2019 están accesibles a través de la plataforma de contenidos a la carta de RTVE.

\subsection{El análisis por modelos}

Cada uno de los modelos de RTV pública se corresponde con un modelo político particular. El modelo pluralista polarizado se caracteriza, como su nombre indica, por tener un sistema político más heterogéneo, con una mayor divergencia y unos medios que tienden más a la opinión. El modelo democrático-corporatista, posee un fuerte pluralismo externo y un sistema de medios con un alto nivel de autonomía. El modelo liberal, por último, y centrándonos particularmente en el caso de la $\mathrm{BBC}$, se corresponde con una larga tradición de independencia y relevancia de los medios públicos y una alta profesionalización. A efectos de esta investigación, la subdivisión regional nos permite tratar las particularidades que tienen los territorios en los que conviven diferentes identidades regionales, como es el caso de Bélgica y la VRT Flamenca, de la cual se pretenden extraer diferencias al compararla con la vecina NPO holandesa, hegemónica hasta hace pocos años en el conjunto de los Países Bajos.

En los dos casos analizados del modelo pluralista polarizado podemos observar importantes cambios en cuanto al número de participantes en los debates electorales televisados, por ejemplo, en el caso de Andorra, pasando de 4 a 7 en las últimas elecciones; y en el caso de España, que pasó del formato 'duelo' complementado por otros sub-debates, a un modelo de debate a 5, adaptándose a los cambios en la representación parlamentaria. Se puede observar como en este caso el modelo pluralista polarizado, más volátil y cambiante, parece afectar al incremento de participantes por el también incremento del número de candidatos con posibilidades de llegar al gobierno.

En el modelo democrático-corporatista, es posible observar cómo tanto la ZDF alemana como la ORF austríaca mantienen tanto el formato como el número de debates estable a lo largo de los últimos 3 períodos electorales, al igual que ocurre con el modelo liberal de la BBC Británica, que tan sólo varía ligeramente en un participante en las elecciones de 2010, pero que ha mantenido el formato en los últimos 2 períodos electorales.

En los dos modelos de la subdivisión regional existe una tendencia a la innovación en paralelo en cuanto al número de participantes y el formato del debate televisivo, sin embargo ambos países han mantenido el debate único para cada uno de los períodos electorales. En este sentido podemos observar que las subdivisiones regionales guardan más similitudes con el modelo pluralista polarizado que con el democrático-corporatista, teniendo en cuenta el número de debates por período electoral y la tendencia a la modificación del número de participantes.

\section{Discusión}

El análisis de las plataformas de VOD de las siete televisiones públicas incluidas en la muestra permite responder las preguntas de investigación y abordar de modo satisfactorio los objetivos planteados. Las preguntas sobre el número debates electorales se ha realizado/emitido en los medios públicos de cada país a lo largo de los últimos años $(P I-1)$ y sobre la variación de formato en cuanto a número de partidos participantes $(P I-2)$ quedan nítidamente respondidas en la tablas resumen aportadas. Hay modificaciones de formato en 5 países, a excepción de Alemania y Austria, y un aumento de participantes en 4 de ellos, a excepción de Alemania, Austria y el Reino Unido. No puede hablarse de una tendencia única, aunque se registran países que casi duplican el número de debates electorales por los medios públicos. 
La pregunta de investigación 1 (PI-3) ¿recogen las plataformas de VOD de las RTV públicas europeas los debates electorales televisados emitidos en sus propios canales? se responde con la constatación de que existe una notable disparidad entre las televisiones públicas que tienen accesibles los debates electorales en sus plataformas de VOD, siendo el caso más común que estos no se encuentren disponibles. En los dos casos del modelo pluralista polarizado, Andorra y España, vemos como existe una cierta tradición de almacenamiento de estos contenidos en las plataformas de VOD, que contienen de una forma organizada y accesible los debates electorales televisados de los últimos 10 años. De la misma manera, parece que en los últimos 3 años comienza a sentirse una tendencia por parte de la NPO y la ZDF, ambos del modelo democrático-corporatista, de permitir el acceso a estos contenidos en sus VOD, siendo visibles los vídeos de los debates de las últimas elecciones federales en ambos canales.

Al identificar cuáles son las tendencias en los formatos de los debates electorales televisados en las RTV públicas de Europa (PI-4) se observa, de manera casi general, que los vídeos de los debates electorales televisados son accesibles a través de otras plataformas, siendo en todos los casos subidos por usuarios ajenos a las televisiones públicas, de manera independiente. Esto sugiere un cierto desinterés por parte de las televisiones públicas europeas en cuanto a mantener una hemeroteca de estos debates televisados, teniendo en cuenta que solo 5 de los 7 ejemplos analizados han sido consistentes a la hora de almacenar estos contenidos. Es notable también el hecho de que en el caso de la ORF austríaca sea más habitual encontrar referencias a elecciones de países más grandes a través de búsquedas genéricas con la palabra 'elecciones federales' a través de sus propios filtros internos de búsqueda.

En cuanto a si existen diferencias o similitudes en estos formatos según el modelo de RTV pública (PI-5) al formato de los debates, en los países sajones (Reino Unido, Alemania, Austria) reina el formato del 'duelo' entre dos candidatos. En el caso de Reino Unido y Alemania este debate siempre tiene lugar entre los dos candidatos con opciones reales de conseguir la presidencia, y en el caso austríaco podemos observar la sublimación de este formato de duelo al repetirse hasta 10 veces realizando todas las combinaciones posibles de candidatos.

Al determinar si existen rasgos comunes o diferencias en el formato de los debates electorales televisados según los 3 modelos de Hallin y Mancini (2004) (PI-6) se ve como en el caso de los países del modelo pluralista polarizado, el formato de debate se adapta a los cambios que van ocurriendo en la realidad política de cada país, cosa que también sucede en el caso de Holanda y Bélgica, marcados por los sub-debates regionales y sus particularidades étnico-políticas, que parecen generar un efecto pluralizador similar a lo que ocurre con sus vecinos del modelo mediterráneo, y por tanto parece que reclaman una mayor diversidad tanto en el formato como en el número de participantes. En los debates televisados de la NPO holandesa se ha optado por una idea innovadora, la de aplicar un mecanismo de co-creación a través de las redes sociales para determinar el formato del debate. Este hecho va en línea con las tendencias que señalan diversos académicos sobre la gestión de productos y contenidos a través de la co-creación con la audiencia, aunque de momento es tan solo un caso aislado entre todas las televisiones analizadas.

Los datos obtenidos del análisis de los VOD describen el dispar uso del debate como formato en siete televisiones públicas y refutan la idea de que estas plataformas se estén utilizando también como hemeroteca de la memoria audiovisual política y, por tanto, como elemento de transparencia y acceso de los ciudadanos a las discusiones políticas más relevantes de cara a la elección de sus representantes. 


\section{Referencias}

Alford, J., Hughes, O. (2008). Public value pragmatism as the next phase of public management. American Review of Public Administration 38(2), 130-148.

Anaya, I. (2014). El análisis del discurso protocolario: elaboración y conceptualización. Estudios filológicos, (54), 7-29.

Bañón, A. M. (1997). La interrupción conversacional. Propuestas para su análisis pragmalingüístico. Analecta Malacitana.

Berrocal Gonzalo, S., Dader García, J. L. y Rospir Zabala, J. (2003). Comunicación política en televisión y nuevos medios. Ariel.

Berrocal, S. 2003. La personalización en la política, en S. Berrocal (coord.), Comunicación Política en Televisión y Nuevos Medios (pp. 55-79). Ariel.

Blas, J. L. (2001). No diga chorradas: La descortesía en el debate político cara a cara. Una aproximación pragma-variacionista. Oralia, (4), 9-45.

Blas, J. L. (2011). Políticos en conflicto. Una aproximación pragmático-discursiva al debate electoral cara a cara. Peter Lang.

Campo Vidal, M. (2013). La cara oculta de los debates electorales: Los debates cara a cara presidenciales en España. Instituto de Comunicación Empresarial (ICE) y Nautebook.

Campos Freire, F., Rodríguez Castro, M. \& de Mateo Pérez, R. (2019). The Trend of Assessment Indicators for Public Service Media in Europe. Communication: Innovation \& Quality, 3-19. https://www.doi.org/10.1007/978-3-319-91860-0_1

Campus, D. 2010. Mediatization and Personalization of Politics in Italy and France: The Cases of Berlusconi and Sarkozy. The International Journal of Press/Politics, 15(2), 219-235.

Canel, M. J. (2006). Comunicación política: Una guía para su estudio y práctica. Tecnos.

Caprara, G. V. 2007. The personalization of modern politics. European Review, 15(2), 151-164.

Carlin, D. P., Howard, C., Stanfield, S., \& Reynolds, L. (1991). The effects of presidential debate formats on clash: A comparative analysis. Argumentation and Advocacy, (27), 126-136.

Casero-Ripollés, A., Feenstra, R. y Keane, J. (2016). La reconfiguración de la democracia: el laboratorio político español. Editorial Comares.

Castells, M. (2009). Comunicación y poder. Alianza.

Coleman, S. (2000). Meaningful political debate in the age of the soundbite. En Coleman, S. (Ed.), Televised election debates. International perspectives. MacMillan Press.

Conde-Vázquez, E., Fontenla-Pedreira, J. y Rúas-Araújo, J. (Eds.) (2019). Debates electorales televisados: del antes al después. Cuadernos Artesanos de Comunicación, (154). Latina. 
Council of Europe (2019). Yearbook 2018/2019. Key Trends. Television, Cinema, Video and OnDemand Audiovisual Services - The Pan-European Picture. European Audiovisual Observatory. https://rm.coe.int/yearbook-keytrends-2018-2019-en/1680938f8e

Díez Nicolás, J. y Semetko, H. A. (1995). La televisión y las elecciones de 1993. Comunicación política. Universitas.

Fernández, F. (2000). Estrategas del diálogo. La interacción comunicativa en el discurso políticoelectoral. Método-Ediciones.

Fuentes, C. (2009). El debate entre Zapatero y Rajoy: ¿convencer a quién? Estudio textual e interactivo. Tonos Digital, (18).

Fields, B., Jones, R. \& Cowlishaw, T. (2018). The Case for Public Service Recommender Algorithms. BBC London, p. 22-24.

Gallego Reguera, M. (2009). El debate de los debates 2008: España y EE. UU. Àmbit.

Gallego Reguera, M. (2012). El debate del debate 2011: España. Dykinson.

Gallego-Reguera, M. y Martínez, I. (2019). ¡Organización!: Producción y realización de los debates electorales en España (1993-2016). En Conde-Vázquez, E., Fontenla-Pedreira, J. y Rúas-Araújo, J. (Eds.), Debates electorales televisados: del antes al después, Cuadernos Artesanos de Comunicación, (154), 67-83. https://doi.org/10.4185/cac154

Hallin, D. \& Mancini, P. (2004). Comparing Media Systems: Three Models of Media and Politics. Cambridge University Press. https://doi.org/10.1017/CBO9780511790867

Herranz-Rubio, C. (2020). La producción científica sobre debates electorales en España: Análisis bibliométrico (1993-2018). Revista Mediterránea de Comunicación/Mediterranean Journal of Communication, 11(1) (En preprint). https://www.doi.org/10.14198/MEDCOM2020.11.1.2

Horváth, T. \& de Carvalho, A. (2017). Evolutionary computing in recommender systems: a review of recent research. Natural Computing, 16(3), 441-462.

Kraus, S. (1962). The Great Debates. Indiana University Press.

Kraus, S. (2000). Televised presidential debates and public policy. Routledge.

Langer, A. I. 2007. A Historical Exploration of the Personalisation of Politics in the Print Media: The British Prime Ministers (1945-1999), Parlamentary Affairs, 60(3), 371- 387.

Lanoue, D. \& J. Schrott, P.R. (1991). The joint press conference. The history, impact and prospects of American presidential debates. Greenwood Press.

Marín, B. (2003). Debates electorales por televisión. En Berrocal Gonzalo, S., Dader García, J. L., y Rospir Zabala, J. Comunicación política en televisión y nuevos medios. Ariel.

Martel, M. (1983). Political campaign debates, images, strategies and tactics. Longman. 
Matera, F. \& Salwen, M. (1996). Unwieldy questions? Circuitous answers? Journalists as panelists in presidential election debates. Journal of Broadcasting and Electronic Media, (40), 309-317.

McKinney, M. S. \& Carlin, D. B. (2004). Political Campaign Debates. En L. L. Kaid, Handbook of Political Communication Research (pp. 203- 234). Lawrence Erlbaum Associates.

Micovic, M. (2014). La comunicación y el discurso políticos en España y Serbia. Análisis comparativo de las estrategias argumentativas utilizadas en los debates electorales televisivos. Tesis doctoral. http://diposit.ub.edu/dspace/handle/2445/62703

Navarrete, F. (2012). Claves para realizar un debate electoral”. En Gallego Reguera, M. (Coord.), El debate del debate 2011: España. Dykinson.

Núñez-Mussa, E. (2018). Variación y distribución de los roles periodísticos en los debates presidenciales televisados de Chile en 2005 y 2013. En Memorias XIV Congreso de la Asociación Latinoamericana de Investigadores de la Comunicación. Grupo Temático 16: Estudios sobre Periodismo. Universidad de Costa Rica.

Ofcom. (2018). Media Nations: UK, 2018. Ofcom Annual Media Nations Report.

Pariser, E. (2011). The troubling future of internet search. The Futurist: World Trends and Forecasts.

Ruiz Contreras, M. (2007). La imagen de los partidos políticos: El comportamiento electoral en España durante las elecciones generales de 1993 y 1996. Centro de Investigaciones Sociológicas (CIS).

Schroeder, A. (2008). The Presidential Debates: fifty years of high-risk TV. Columbia University Press.

Schroeder, A. (2012). Los formatos de los debates televisivos. En Gallego Reguera, M. (Coord.), El debate del debate 2011: España. Dykinson.

Schroeder, A. (2016). Presidential debates. Risky business on the campaign trail. Columbia University Press.

Simonofski, A., Snoeck, M. \& Vanderose, B. (2019). Co-creating e-Government Services: An Empirical Analysis of Participation Methods in Belgium, In Setting Foundations for the Creation of Public Value in Smart Cities, (pp.225-245). https://doi.org/10.1007/978-3-319-98953-2_9

Sørensen, J. \& Hutchinson, J. (2018). Algorithms and public service media. Public Service Media in the Networked Society RIPE, 91-106.

Stepp, C. S. (1987). The role of journalist's questions in campaign forums. Political Communication and Persuasion, (4), 279-288.

Túñez-López, José-Miguel; Toural-Bran, Carlos; Cacheiro-Requeijo, Santiago (2018). Uso de bots y algoritmos para automatizar la redacción de noticias: percepción y actitudes de los periodistas en España. El profesional de la información, 27(4), 750-758. https://doi.org/10.3145/epi.2018.jul.04 
Túñez-López, J. M.; Toural-Bran; C. y Valdiviezo-Abad, C. (2019). Automatización, bots y algoritmos en la redacción de noticias. Impacto y calidad del periodismo artificial. Revista Latina de Comunicación Social, (74), 1.411- 1.433.

Turcotte, J. (2015). The news norms and values of presidential debate agendas: an analysis of format and moderator influence on question content. Mass Communication and Society, 18(3), 239-258.

Vidal Riera, F. (1997). Los debates "cara a cara": Fundamentos básicos para la celebración de debates electorales audiovisuales entre los líderes de los partidos mayoritarios (Tesis Doctoral). Universidad Complutense de Madrid: Madrid.

\section{AUTORES:}

\section{Martín Vaz-Álvarez}

Periodista graduado por la Universidad de Santiago de Compostela, máster en Investigación en Comunicación Audiovisual y Periodismo por la Universidad Autónoma de Barcelona y doctorando del Programa de Doctoramiento en Información y Comunicación Contemporánea de la Universidad de Santiago de Compostela. Su tesis está enfocada en la co-creación en las radiotelevisiones públicas, la innovación y las nuevas tecnologías. Actualmente forma parte del grupo de investigación Novos Medios del Departamento de Ciencias de la Comunicación de la Universidad de Santiago de Compostela como investigador predoctoral en formación, siendo beneficiario de una ayuda de Formación de Profesorado Universitario (ref. FPU19/06204)

martin.vaz.alvarez@usc.es

\section{Índice H: NA}

Orcid ID: http://orcid.org/0000-0002-4848-9795

Google Scholar: $\underline{\text { https://scholar.google.es/citations?hl=es\&user=Dj9HHNEAAAAJ }}$

\section{José Miguel Túñez-López}

Profesor Titular, acreditado Catedrático y director de la Escuela de Doctorado Internacional en Artes y Humanidades, Ciencias Sociales y Jurídicas de la Universidad de Santiago de Compostela. Actualmente está enfocando su investigación en el ámbito de la automatización en medios de comunicación.

miguel.tunez@usc.es

\section{Índice H: 19}

Orcid ID: https://orcid.org/0000-0002-5036-9143

GoogleScholar: https://scholar.google.es/citations?user=fGFou0MAAAAJ\&hl=es

\section{Carmen Costa-Sánchez}

Profesora de Comunicación Corporativa en el Grado en Comunicación Audiovisual de la Universidade da Coruña. Doctora en Comunicación por la Universidade de Santiago de Compostela. Premio Extraordinario de Doctorado por la Facultad de Ciencias de la Comunicación de la USC. Sus líneas de investigación fundamentales son la Comunicación Corporativa, la Comunicación para la Salud, la Comunicación móvil y transmedia. Es miembro del Grupo de Investigación en Cultura y Comunicación Interactiva de la Universidade da Coruña.

carmen.costa@udc.es

\section{Índice H: 19}

Orcid ID: https://orcid.org/0000-0001-8154-9537

Google Scholar: https://scholar.google.es/scholar?hl=es\&q=Carmen+Costa-Sánchez\&btnG=\&lr= 